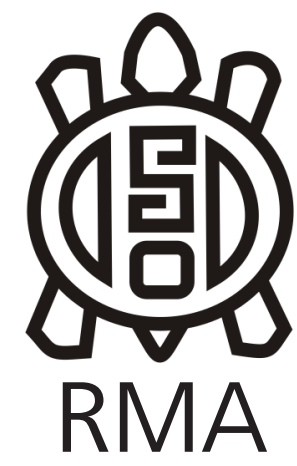

Arqueología

\title{
Tomar la fábrica, recuperar la historia. Memorias, paisajes y patrimonios en un frigorífico recuperado (Bahía Blanca, Argentina)
}

\author{
Take the factory, recover history. Memories, landscapes and heritage in \\ a recovered Cold Store (Bahía Blanca, Argentina) \\ Alejandra Pupio * Hernán Tomassini **
}

*Departamento de Humanidades (Universidad Nacional del Sur), Comisión de Investigaciones Científicas, Buenos Aires E-mail: mapupio@uns.edu.ar

${ }^{* *}$ CONICET-Departamento de Humanidades (Universidad Nacional del Sur). E-mail: tomassinihernan@gmail.com

\begin{abstract}
Resumen
Este trabajo analiza los procesos de memoria y patrimonialización de un conjunto de bienes históricos y arqueológicos del siglo XIX, localizados en el predio del Frigorífico INCOB, empresa recuperada en la ciudad de Bahía Blanca. Tiene como objetivo analizar las múltiples lecturas del espacio desde la perspectiva de los y las trabajadoras de la fábrica, para comprender los sentidos, las narrativas, las formas de clasificarlo y definirlo históricamente desde su presente. En este sentido, en primer lugar, se presentan las distintas capas de historicidad que habitan el espacio analizado y que dieron lugar a los distintos paisajes culturales. En segundo lugar, se analizan las percepciones de las y los trabajadores sobre estos bienes históricos y arqueológicos que forman parte de su paisaje cotidiano. Finalmente, se discute a partir de este caso de estudio las concepciones heterogéneas acerca del patrimonio, los planes y las propuestas de gestión de la comunidad del frigorífico, los funcionarios municipales y los investigadores.
\end{abstract}

Palabras clave: Memorias; Paisaje; Patrimonio; Arqueología; Comunidades locales.

\begin{abstract}
This work analyses the processes of memory and heritage of a set of historical and archaeological assets of the nineteenth century, located on the site of the INCOB Cold Store, a recuperated company in the city of Bahía Blanca. It aims to analyze the multiple readings of the space from the perspective of the workers of the recovered factory, to understand the meanings, the narratives, the ways to classify it and define it historically from its present. In this sense, in the first place, the different layers of historicity that inhabit the examined space, and that gave rise to the different cultural landscapes are presented. Secondly, the perceptions of workers about these historical and archaeological assets that are part of their daily landscape are analyzed. Finally, the heterogeneous conceptions of heritage, plans, and management proposals of the INCOB community, municipal officials, and researchers are discussed from this case study.
\end{abstract}

Keywords: Memories; Landscape; Heritage; Archaeology; Local communities.

\section{Introducción}

Este trabajo se suma a las producciones acerca de los procesos de memoria y patrimonialización desde la perspectiva del conocimiento situado (sensu Haraway, 1995), que tiene en cuenta las contingencias espaciales y temporales en las que se producen los saberes y las tomas de decisiones. En el contexto de ciudades diversas y desiguales, como Bahía Blanca (provincia de Buenos Aires), se evidencian las dificultades de un tratamiento homogéneo acerca del patrimonio local. Por el contrario, cada vez con mayor profundidad, los casos de estudio y la producción de teoría señalan que esta categoría debe ponerse en discusión y considerar los vínculos entre las memorias, los materiales y los lugares para observar en cada caso las conceptualizaciones que elaboran los diferentes colectivos sociales y políticos (Crespo, 2010; Corimayo y Acuto, 2015; Endere, 2016; Flores y Acuto, 2015; Millán, Chaparro y Mariano, 2019; Rivolta, Montenegro, Menezes y Nastri, 2014).

Es así, que más allá de las dificultades para una definición, 
entendemos al patrimonio local (situado) como un concepto y un conjunto de prácticas que emergen de la modernidad del siglo $X X$ y que no necesariamente son compartidas por todos los miembros de las comunidades locales, regionales y nacionales. Incluso, esta categoría puede estar vacía de contenido y los bienes o prácticas suelen ser definidas o clasificadas con otras lógicas sociales o identitarias. Poner el foco en estos temas conlleva a analizar los modos en que opera la producción del pasado y su materialidad en el paisaje cultural, teniendo en cuenta que esto siempre ocurre en un escenario de tensión entre los poderes hegemónicos y las resistencias contra-hegemónicas.

En esta investigación, el análisis se centra en la producción de conocimiento sobre el pasado y en la toma de decisiones sobre un conjunto de bienes culturales por parte de las y los trabajadores/as de una fábrica recuperada en el área sur de la ciudad de Bahía Blanca, lindantes al barrio Villa Nocito. En los terrenos que actualmente ocupan las instalaciones del Frigorífico recuperado INCOB (Industria de la Carne Obrera Bahía Blanca) fueron localizados un rasgo, la denominada "zanja de Rosas", y un edificio familiar conocido como "casa Pronsato" o estancia "La Catalina" (Figura 1), ambas construcciones del siglo XIX. Este sector de la ciudad es donde se encuentran algunos de los barrios con menor acceso a las políticas públicas relativas a la salud, educación e infraestructura. Como consecuencia, las cuestiones relativas al patrimonio cultural en estos espacios, no recibieron la atención que la gestión municipal provee a otras áreas del ejido urbano.

En el predio del frigorífico, el presente habita y produce una espacialidad que tiene semejanzas a otros procesos de recuperación de fábricas que se produjeron a fines de la década de 1990 y especialmente como consecuencia de la crisis de 2001. Como señalan Rebón (2005) y

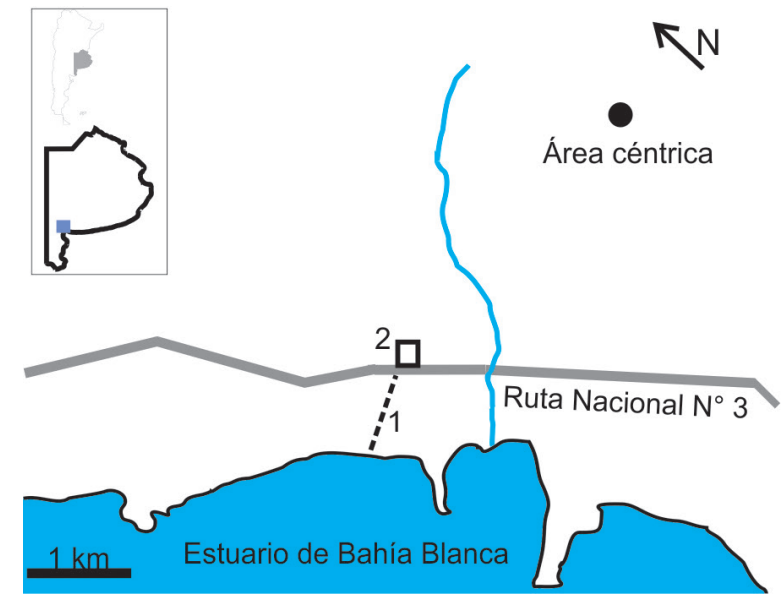

Figura 1. Área de estudio. 1. Localización de la denominada "zanja de Rosas"; 2. Localización de la casa-azotea propiedad de Domingo Pronsato.

Figure 1. Study area. 1. Location of the so-called "Rosas" ditch ("zanja de Rosas"); 2. Location of the roof-house owned by Domingo Pronsato.
Kasparian (2013), son espacios caracterizados por la lucha obrera para la recuperación de sus fuentes de trabajo de forma autogestiva y, al mismo tiempo, la construcción de relaciones de reciprocidad no mercantiles con los vecinos de la ciudad, con instituciones sociales, políticas y la sociedad en general. Estas dobles articulaciones espaciales formaron parte de la estrategia política que algunas empresas recuperadas se dieron para construir legitimidad social y apoyo comunitario, e incluyen la creación de escuelas y centros culturales, entre otras actividades (Kasparian, 2013). Esto caracteriza los vínculos y acciones de las y los trabajadores/as del frigorífico INCOB, con un fuerte impacto y articulación con las instituciones bahienses, entre las que se destaca la Universidad Nacional del Sur (UNS).

Desde este lugar, se busca analizar las múltiples lecturas de la espacialidad desde la perspectiva de las y los trabajadores de la fábrica recuperada, para entender los sentidos, las narrativas sobre los bienes culturales presentados, así como las formas de clasificarlos y definirlos históricamente desde su presente. De este modo, el primer objetivo es comprender las distintas capas de historicidad que habitan el espacio analizado y que dieron lugar a los distintos paisajes culturales. Por otro lado, se propone presentar las percepciones que la comunidad de trabajadoras y trabajadores construye de estos bienes que forman parte de su paisaje cotidiano. Finalmente, se discute a partir de este caso de estudio las concepciones heterogéneas acerca del patrimonio y los planes y propuestas de la comunidad del frigorífico, los gestores del patrimonio y los investigadores/as. Para esto se trabaja dentro del marco de la Arqueología del Paisaje que, como indica Acuto (2013), permite analizar la espacialidad de la modernidad capitalista, entendiéndola como una superposición de paisajes y experiencias espaciales que incluyen los de control, vigilancia, de producción (Zukin, 1991) y resistencia (Pile y Keith, 1997).

El paisaje como una especie de cultura material puede ser reconstruido tomando la metáfora biográfica que propone Kopytoff (1991) para los objetos (Bloemers, 2010). Estas biografías se reconstruyen desde múltiples fuentes y con una perspectiva interdisciplinar que incluye, entre otros, a los campos de la Arqueología, la Historia y del patrimonio. Desde este último campo de estudio, esto es relevante ya que la presencia de capas de sentido en el espacio no solo tiene una dimensión diacrónica sino también sincrónica. De este modo, las transformaciones anteriores pueden leerse en su efecto continuo sobre el entorno contemporáneo y por medio del palimpsesto de objetos y estructuras producidos en ese proceso. Por otro lado, la biografía del paisaje representa el carácter multivocal del pasado y del presente. Los individuos y las sociedades poseen diversas memorias de la historia regional, le atribuyen significados, la ordenan, la clasifican y construyen narraciones sobre ella (Ricoeur, 1995). Esas 
memorias necesariamente están modeladas por el marco social y por lo tanto participan de las disputas materiales e ideológicas de una comunidad particular (Halbwachs, 2004; Jelin, 2002). La memoria, por otro lado, comparte muchas características con el patrimonio, ya que en ambas dimensiones se crean sentidos a través de la construcción de narrativas, de acciones e intervenciones en el espacio público atravesadas por las relaciones desiguales de poder. Es por eso que ambos son parciales, subjetivos, controvertidos, políticos y sujetos a una historia particular que cambia dinámicamente. Quizá, como señala Sather-Wagstaff (2015), lo más importante de comprender es que el patrimonio, entendido como un elemento social y construcción discursiva, no puede existir sin memoria en acción en el mundo cotidiano. Si la relación con el pasado está marcada por los recuerdos de los grupos sociales que articulan sus identidades en el presente, entonces la memoria y el trabajo de la memoria son propiedades intrínsecas y constitutivas del patrimonio.

Para tratar estos temas se desarrolló una metodología cualitativa que combinó diferentes técnicas para la recolección de datos, lo que facilitó una comprensión más profunda de la complejidad social en torno a los bienes. Esto permitió analizar la historia del paisaje integrando la información arqueológica y el análisis cartográfico y documental. Por otro lado, las observaciones realizadas en distintas acciones públicas organizadas en la fábrica recuperada y la realización de un grupo focal con trabajadoras y trabajadores permitieron identificar las experiencias de diferentes actores y confrontarlas con las de otros que incluyen a los funcionarios municipales a cargo de la gestión del patrimonio e investigadores. Cada uno con sus saberes y sus prácticas, tienen el desafío de interactuar para participar en distintas acciones que pongan en discusión las memorias acerca del pasado representado en esa cultura material y sobre las decisiones, las conceptualizaciones e interpretaciones sobre estos bienes.

\section{Tiempo y espacio construido}

El área de estudio se encuentra en el sector sudoeste de la ciudad, ubicada en el límite entre la zona industrial y diversos barrios residenciales. Este espacio fue objeto de la expansión territorial en la primera mitad del siglo XIX como consecuencia de la instalación de la Fortaleza Protectora Argentina en 1828, enclave militar que dio origen posteriormente a la ciudad de Bahía Blanca (Ratto y Santilli, 2004). Entre los años 1833 y 1834 tuvo lugar la denominada "Expedición al Desierto" comandada por Juan Manuel de Rosas, cuyo objetivo fue el adelantamiento de la línea fronteriza hasta el río Negro y la incorporación de tierras al estado provincial. Esta estrategia de avance militar produjo una significativa modificación en el diseño espacial de la localidad, al ampliarse el trazado del ejido urbano alrededor de la fortaleza con la incorporación de nuevas manzanas y terrenos (Ockier, 2001). Así mismo, se ampliaron y consolidaron los elementos defensivos, construyéndose dos zanjas integradas, ubicadas a una distancia de entre 700 y 4000 m sobre los sectores noreste y sudoeste de la fortaleza. Junto a estas zanjas, se erigieron, además, cuatro pequeños fortines distribuidos en diferentes puntos de su recorrido, denominados "No1", "N²", "N³" y "N4" (Pronsato, 1954; Puig Domenech y Thill, 2003; Puliafito, 2007, entre otros). El diseño defensivo permitió, en teoría, mantener protegido al poblado, en tanto cada una de las zanjas finalizaba su recorrido articulándose con elementos naturales del ambiente: el arroyo Maldonado y el estuario, por un lado, y el arroyo Napostá por otro (Figura 2).

Desde la segunda mitad del siglo XIX el área de estudio fue modificándose de manera continua como consecuencia de la definición de los límites del partido de Bahía Blanca en 1865 y de la conformación de un mercado inmobiliario que produjo el traspaso de las tierras públicas a manos privadas (Bayón y Pupio, 2003). Como consecuencia de esta expansión, se otorgaron
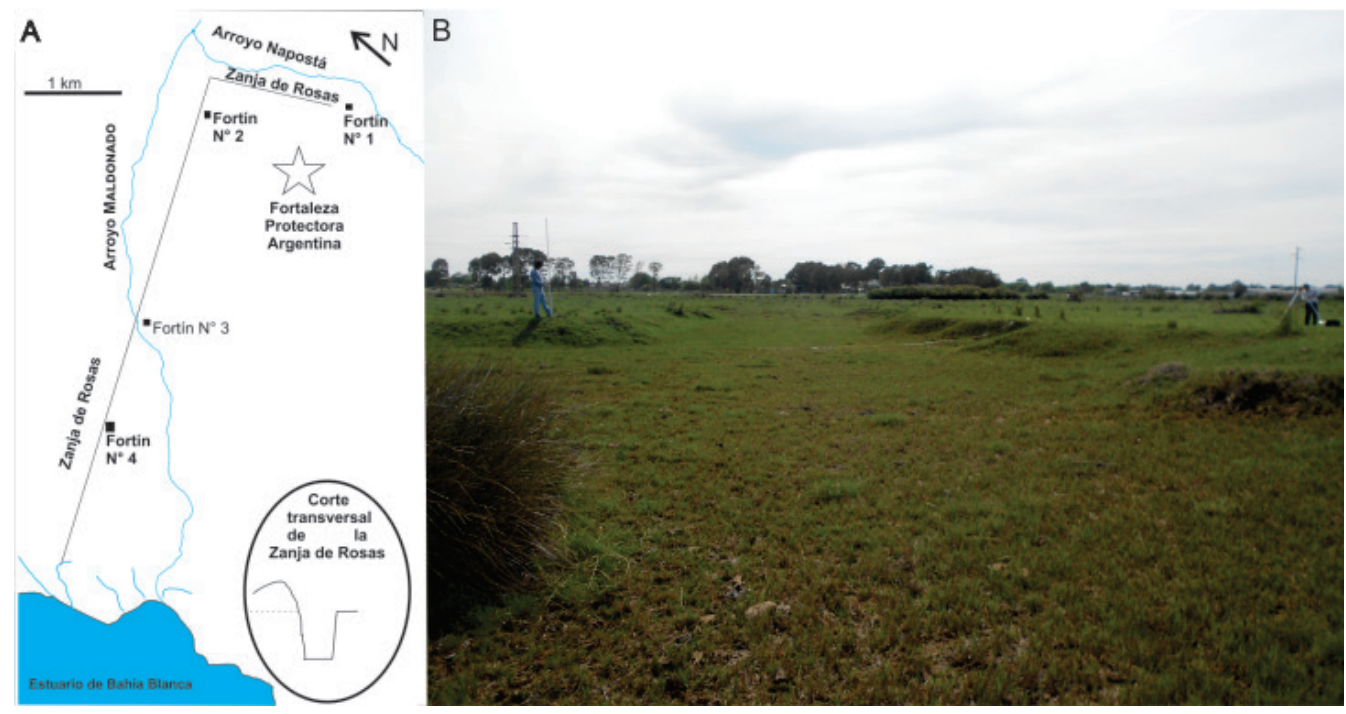

Figura 2. A: Plano del trazado original de la zanja (Modificado de Pronsato, 1954); B: Vista de la denominada "zanja de Rosas".

Figure 2. A: Plan of the original ditch layout (Modified from Pronsato, 1954); B: View of the so-called "Rosas" ditch. 
parcelas de tierras a particulares ubicadas dentro del ejido del partido hasta la margen izquierda del río Sauce Chico (Pupio y Perriére, 2013). Entre estas ocupaciones se encuentra la chacra $N^{\circ} 100$ propiedad de Domingo Pronsato (destacado intelectual, político y artista de la ciudad). En la década de 1870 adquirió una propiedad donde construyó el edificio de la casa-azotea de la que se hace referencia en este trabajo, erigida sobre la ubicación anterior del fortín $\mathrm{N}^{\circ} 4$. Esta casa responde a una tipología constructiva presente en la provincia de Buenos Aires en el siglo XIX. También denominadas reductos rurales fortificados, combinaban características productivas y defensivas, y se caracterizaban por la presencia de una planta habitacional superior que permitía tanto el control del paisaje como la defensa ante un eventual ataque, tanto de grupos indígenas como de otros miembros de la sociedad hispano-criolla (Pastrana, 1965) (Figura 3). Se deriva de estas características que el paisaje construido fue la respuesta a una estrategia de poder coercitivo, con el esfuerzo puesto en el establecimiento de una línea espacial efectiva para excluir y/o restringir el acceso de los indígenas. Esto dio origen a un paisaje complejo compuesto por instalaciones extractivo-productivas (casas azoteas), militares- defensivas (fortines, zanjas) y comerciales (pulperías) (Bayón y Pupio, 2003). Hasta la década de 1870 este era un paisaje de frontera caracterizado por el conflicto y la tensión entre lógicas distintas de conceptualización del espacio. En este contexto, la frontera fue vista como el límite que separaba a las sociedades blancas de las indígenas (Mandrini, 1992).

Hacia fines del siglo XIX y principios del XX se produjo otra etapa de intervención en el área de estudio con los cambios que imprimió la empresa Bahía Blanca Noroeste (F.C.B.B.N.O). Estas modificaciones implicaron el tendido de rieles y la infraestructura asociada al mantenimiento de locomotoras, como el denominado galpón "Maldonado", a lo que se sumó la construcción de viviendas particulares para los trabajadores (Guerreiro, 2011; Miravalles, 2013). Lindante a este sector se loteó la Chacra 24, que dio origen a Villa Nocito, constituyéndose como un barrio de características obreras (Bianchi Díaz y Ockier, 2012).

A partir de la segunda mitad del siglo XX el trazado del área se vio modificado por la realización de obras de infraestructura y por la llegada de una corriente migratoria proveniente de países limítrofes principalmente de Chile, aunque también, en menor medida, de Bolivia y Paraguay. La importante proporción de habitantes chilenos, o descendientes, permitió que este barrio sea reconocido territorialmente como colectivo de dicho país en Bahía Blanca (Diez, 1999; Ockier et al., 2007). En términos generales, este barrio, así como otros de la zona sur, se caracterizan por la ausencia de políticas públicas que garanticen el acceso a la educación, la salud, el trabajo, los servicios y la falta de planificación territorial (Prieto, 2012). Finalmente, Villa Nocito puede ser caracterizado como un barrio de trabajadores, con una larga historia de militancia y organización política y social desde la década de 1960, experiencias que lo dotaron de un fuerte componente comunitario (Dominella, 2015). A pesar de la persecución iniciada a muchos de los militantes, la dictadura militar (1976-1983) no logró desarticular estas redes sociales $y$, con el retorno de la democracia, se conformaron
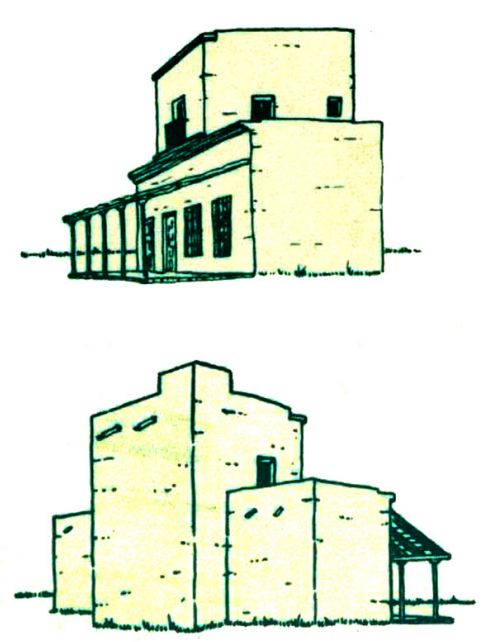

A

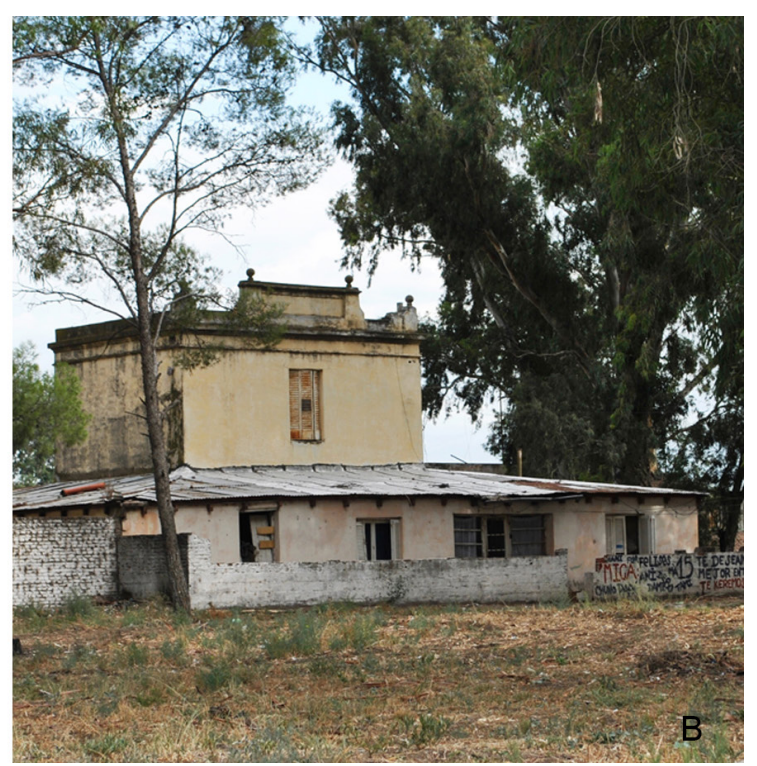

Figura 3. A. Estructura arquitectónica de las denominadas casas-azoteas o reductos rurales fortificados (Pastrana, 1965); B. Casa azotea en el predio del frigorífico INCOB (Fotografía de los autores).

Figure 3. A: Architectural structure of so-called roof-houses or fortified rural redoubts (Pastrana, 1965); B: Roof-house on the property of the INCOB Cold Store. 
distintos espacios de militancia política, social y cultural como la Sociedad de Fomento, la Biblioteca Popular Barrial Gabriela Mistral, el Colectivo Periférico Cultural y la Murga La Periférica. En este contexto barrial se encuentra el frigorífico INCOB donde está localizada la casa azotea. Se trata de una empresa recuperada por las y los trabajadores/as como consecuencia de la declaración de quiebra de la firma Guillermo Paloni e Hijos en el año 2005. El proceso de recuperación implicó nuevos vínculos de las y los trabajadores/as con la comunidad barrial y bahiense, entre los que se destaca los vínculos con la UNS. Este espacio adquiere ahora características nuevas derivadas del proceso de toma y recuperación de la empresa por parte de las y los trabajadores/as, análisis que será retomado en los próximos acápites.

\section{La frontera como tropos fundacional de los pueblos bonaerenses}

El punto de partida de este trabajo fue la acción de vecinos de la ciudad quienes en el año 2014 solicitaron al Instituto Cultural de la Municipalidad que la casa azotea y la "zanja de Rosas" sean declarados bienes patrimoniales para su conservación y difusión. A partir de esto se planificaron una serie de acciones que incluyeron los trabajos arqueológicos a cargo de uno de los autores, la búsqueda documental y el trabajo en territorio a partir de los Proyectos de Extensión que una de las autoras coordinó en Villa Nocito desde el año 2013.

Las noticias de la existencia de la "zanja de Rosas" y de una casa azotea, que fuera la chacra de la familia Pronsato y sede de la Observación Astronómica del tránsito de Venus en el año 1882, eran conocidas en las publicaciones locales desde mediados del siglo XX, especialmente por las referencias y fotografías difundidas por Domingo Pronsato, nieto del propietario original de la casa (Pronsato, 1954). Sin embargo, los gobiernos municipales carecieron, al menos hasta la década de 1980, de políticas de gestión patrimonial por lo cual no fueron objeto de atención hasta el momento. Esto no significa que estos bienes no fueran significativos, por el contrario, se constituyeron en foco de interés desde la primera mitad del siglo XX, alrededor de la actividad del Archivo Histórico (1933) y del Museo Histórico y de Ciencias Naturales (1951). Los bienes considerados históricos estaban en relación con las historias escritas por los aficionados locales que recuperaron documentos y realizaron relevamientos en el terreno para reconstruir la historia del siglo XIX, especialmente la relativa a la construcción de la Fortaleza Protectora Argentina y de la expansión militar en la frontera. Como consecuencia de esta acción local y en el contexto de creación de la Comisión Nacional de Museos y Monumentos Históricos (1940), la ciudad contó tempranamente con un Monumento Histórico Nacional en 1944, que fue el denominado Fortín Cuatreros (Pupio y Perriere, 2013).
En estas historias de aficionados locales, las acciones militares fueron presentadas como el punto de origen de la civilización que habría de completarse con la llamada Conquista del desierto (1879).

Esta matriz histórica se consolidó con la creación de la Comisión de Reafirmación Histórica en 1976, organismo encargado de señalizar en el espacio público aquellos acontecimientos o bienes arquitectónicos considerados relevantes. Desde su creación hasta la fecha se recrearon especialmente sitios y eventos de la etapa fundacional, de la Campaña del Desierto y, en menor medida, aquellos representativos de la expansión del modelo agroexportador (Borgani, 2007). Los miembros de esta Comisión contaron con el apoyo institucional de la Municipalidad y con la difusión de sus actividades, propuestas y artículos en el diario local La Nueva Provincia. El periódico tuvo el monopolio de la información y esto se evidenció en la capacidad de reproducir las representaciones y versiones de la historia y del patrimonio vinculadas con la historia militar decimonónica, difundidas en sus páginas por historiadores aficionados, militares y civiles, muchos de los cuales eran miembros de aquel organismo.

En el año 1991, al conmemorarse el 163 aniversario de la ciudad, aquella Comisión emplazó una señalización sobre

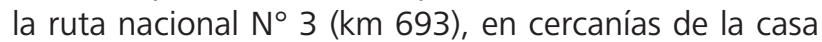
azotea. Su texto indicaba: "A 200 ms al N.E se levanta el fortín 'La Catalina', construido en el año 1833 por el T. Cnel. Antonio Manuel de Molina, para defensa de ataques indígenas". Con la presencia de autoridades municipales, militares y concejales, el discurso central estuvo a cargo de Desiderio Lagier, miembro de la Comisión de Reafirmación Histórica, quien expresó que este reconocimiento profundizaría el espíritu de argentinidad, el criollismo y la valoración de la figura de Juan Manuel de Rosas, a lo que se sumaba el homenaje a "aquellos brazos anónimos que cavaron la denominada 'zanja de Rosas'". Por su parte, el intendente Jaime Linares, de extracción radical, expuso en su discurso el contrasentido de esta referencia histórica en el momento político vivido al iniciar con una pregunta "¿por qué la Catalina? Es una forma de entender hacia dónde vamos y de dónde venimos. Responde a un modelo de país discutible u objetable, pero con una vocación de avance bien definida" (La ciudad conmemoró..., 1991). Quizá como consecuencia de esta contradicción, esta señalización no fue repuesta luego de haber sido sustraída poco tiempo después.

Si bien estas prácticas de señalización continúan hasta el presente, luego del retorno de la democracia se vislumbró una nueva percepción en el tratamiento de los bienes patrimoniales. En 1984 se creó la Comisión Asesora Permanente ad Honorem para la Preservación del Patrimonio Arquitectónico Urbanístico e Histórico del Partido de Bahía Blanca, integrada por distintas instituciones de la ciudad. Funciona en la municipalidad y tiene como misión exclusiva evaluar los valores 


\begin{tabular}{|c|c|c|}
\hline $\begin{array}{c}\text { Bienes muebles de valor } \\
\text { patrimonial } \\
\text { (Total }=28)\end{array}$ & $\begin{array}{l}\text { Bienes inmuebles de valor } \\
\text { patrimonial } \\
\text { (Total=173) } \\
\text { - Religioso } \\
\text { - } \quad \text { Militar } \\
\text { - Civil de función pública } \\
\text { Civil de función privada }\end{array}$ & $\begin{array}{l}\text { Áreas de valor patrimonial } \\
\text { (Total }=14)\end{array}$ \\
\hline Ferrocarril (casillas, barreras) & Comercios e industrias (24) & Centro histórico \\
\hline $\begin{array}{l}\text { Servicios públicos (farolas, } \\
\text { transformadores, tableros de } \\
\text { electricidad y puentes) }\end{array}$ & Edificios educativos (15) & Areas ferroportuarias (4) \\
\hline $\begin{array}{l}\text { Muebles arquitectónicos (kioscos } \\
\text { art noveau, balcones) }\end{array}$ & $\begin{array}{l}\text { Edificios de arquitectura } \\
\text { ferroportuaria (12) }\end{array}$ & $\begin{array}{l}\text { Conjunto de viviendas } \\
\text { ferroviarias (2) }\end{array}$ \\
\hline \multirow[t]{3}{*}{ Monumentos (8) } & $\begin{array}{c}\text { Edificios de la administración } \\
\text { pública, financiera, religiosa y } \\
\text { prensa (36) }\end{array}$ & Parques de la ciudad (2) \\
\hline & $\begin{array}{c}\text { Edificios de instituciones culturales } \\
\text { y deportivas (12) }\end{array}$ & $\begin{array}{c}\text { Segmentos de las avenidas } \\
\text { principales (Av. Alem y Av. } \\
\text { Colón) }\end{array}$ \\
\hline & Edificios de viviendas familiares (74) & $\begin{array}{c}\text { Barrios de viviendas } \\
\text { familiares residenciales (3) }\end{array}$ \\
\hline
\end{tabular}

Tabla 1. Cantidad y tipo de bienes muebles, inmuebles y áreas de valor patrimonial incorporados al Registro Municipal para la Preservación del Patrimonio Arquitectónico y Urbano desde el año 1994 en el Partido de Bahía Blanca, confeccionada por los autores.

Table 1. Quantity and type of movable and immovable property, and areas of heritage value incorporated into the Municipal Register for the Preservation of Architectural and Urban Heritage (Registro Municipal para la Preservación del Patrimonio Arquitectónico y Urbano) since the year 1994 in the Bahía Blanca district, made by the authors. arquitectónicos de los edificios, por lo cual la mayor parte de sus miembros son arquitectos. En 1994, esta misma comisión presentó la creación del Registro Municipal para la Preservación del Patrimonio Arquitectónico y Urbano que incorporó como patrimonio a un conjunto de bienes inmuebles, muebles y áreas de valor patrimonial referentes de la arquitectura ferroportuaria, edificios de vivienda familiar, públicos y comerciales del área céntrica de fines del siglo XIX y principios del XX (Tabla 1).

Lo antes dicho, permite señalar los límites de la práctica de la gestión patrimonial de las instituciones abocadas a ello. A pesar de la ampliación del concepto de patrimonio dado por las acciones de gestión a partir de la década de 1980, casi todos los bienes declarados de valor histórico están relacionados con aquellos ubicados en el eje de la denominada segunda fundación de Bahía Blanca (fines del siglo $X I X)$, y que se concentran en el área del centro histórico y la ruta ferroportuaria (Ribas y Tolcachier, 2012). Quedan fuera del interés elementos materiales muebles o inmuebles que puedan encontrarse en otros espacios del partido de Bahía Blanca como los aquí descriptos. A pesar de lo cual, eventualmente emerge el interés en estos bienes por acción de vecinos.

\section{La "zanja de Rosas" y la casa azotea desde la arqueología}

La expansión urbana provocó la casi total desaparición del sistema defensivo originalmente diseñado en la primera mitad del siglo XIX, con excepción del tramo de la "zanja de Rosas" y la casa azotea descripta. Con respecto a la zanja, ésta sufrió un proceso de cegamiento durante gran parte del siglo XIX y XX, al quedar cubierta casi en su totalidad por las construcciones del ejido urbano bahiense. Sólo se conservó un tramo, de aproximadamente 700 m, situado entre el estuario y el km 693 de la Ruta Nacional $N^{\circ} 3$ construida en la década de 1940. Este segmento se encuentra modificado por diversos procesos postdepositacionales, tanto naturales como culturales. Por tratarse de un terreno dedicado al pastoreo de ganado, la zanja es alterada constantemente por el pisoteo y tránsito de animales (principalmente bovinos), por lo que se evidencia un constante desmoronamiento de sus bordes. Asimismo, en los sectores más cercanos a la línea de costa, la estructura se ve afectada por las mareas, lo que produce anegamiento y presencia de colonias de cangrejos. Sobre este sector se llevaron a cabo diversos trabajos arqueológicos como la realización de perfiles sedimentológicos y mediciones altimétricas que permitieron conocer sus principales características constructivas y de conservación (Tomassini y Vecchi, 2015; Tomassini, 2017). En el caso de la estancia "la Catalina", esta sufrió diversas transformaciones desde su construcción como casa azotea. Debido a que ha sido utilizada como vivienda hasta la actualidad, fueron modificados sus rasgos arquitectónicos internos. Por este motivo no se han podido realizar actividades, aunque se encuentra planificado realizar prospecciones y sondeos en sus alrededores.

En el contexto de la investigación arqueológica pudieron reconstruirse diferentes aspectos históricos relacionados con la casa de la familia Pronsato. La literatura histórica local señalaba que en esta propiedad se había efectuado la observación astronómica del paso de Venus en 1882 por parte de una Comisión Científica franco-alemana (Peter, 1884). Si bien el objetivo inicial del proyecto 
fue identificar el sitio donde se realizó la observación astronómica, recientemente se ha publicado un trabajo que indica que el lugar de la observación no fue en la casa azotea que aquí se analiza sino en otra edificación similar situada $1500 \mathrm{~m}$ hacia el este, también propiedad de la familia Pronsato (Milesi y Vazquez, 2013). Esta información fue reproducida en el diario local, un año después de su publicación académica, aunque como en muchos otros casos, se sigue reproduciendo la versión de la historia amateur local (Minervino, 2014).

En este punto es importante remarcar que las autoridades vinculadas a la gestión del patrimonio reconocen los valores históricos de estos bienes, a pesar de lo cual consideran que, en el caso de la casa azotea, esta no cuenta con las condiciones edilicias para su declaración como bien inmueble (Comisión Asesora Permanente ad Honorem para la Preservación del Patrimonio Arquitectónico Urbanístico e Histórico del Partido de Bahía Blanca, com.pers.). En esta situación, los autores propusieron en el año 2014, durante las etapas iniciales de este trabajo integrar ambos bienes en un área de valor patrimonial (Ordenanza N 7959, 1994), categoría que incluye "zonas o sitios considerados de interés porque recuerdan los modos de vida característicos de la historia de la comunidad" (Viñuales y Zingoni, 1990).

Ante este y otros casos, las respuestas administrativas a las demandas de vecinos son lentas, ya que se observa un estancamiento en la declaración de bienes a proteger y, al mismo tiempo, se evidencia una falta de coordinación de normas que permitan articular acciones. Esta débil presencia de políticas públicas está acompañada por una escasa participación ciudadana que reclame por el estado de conservación y puesta en valor de los bienes. En la ciudad hubo algunas excepciones, como los reclamos públicos por la demolición de un edificio en el que actualmente funciona un local de comidas rápidas (Tolcachier, 2018). Parte de los problemas observados localmente se deben a una desarticulación de las políticas de ordenamiento territorial y las prácticas integrales de gestión patrimonial que tienen lugar en ese mismo espacio. En este escenario adquiere sentido volver sobre las percepciones que las y los trabajadores/as del frigorífico tienen como protagonistas de ese espacio ya que ninguna política de gestión sobre los bienes en cuestión puede consolidarse sin la participación de la comunidad de la que forman parte.

\section{"Recuperar, tomar, recuperar un frigorífico recuperado"}

En el año 2005 cuando la empresa Paloni S.A. presentó quiebra, 45 trabajadores directos quedaron sin trabajo y tomaron la decisión colectiva de ocupar la fábrica a la espera de su expropiación y control. La toma duró dos años y con ellos la merma de compañeros y compañeras ya que, de 40 iniciales, solo 18 quedaron al conformar la cooperativa. Finalmente, en el año 2007 se aplicó la Ley de Expropiaciones que le permitió a INCOB comenzar a funcionar. A las dificultades propias de cualquier proceso de recuperación se sumó, en este caso, la denuncia por enriquecimiento fraudulento que realizaron las y los compañeros en el año 2015 al primer Presidente de la Cooperativa. Después de una etapa de conflicto, lograron recuperar el frigorífico, expulsar al denunciado e iniciar las acciones legales en su contra, que culminaron, en noviembre de 2019, con una condena de dos años de prisión condicional. Luego de lo que denominan la "refundación" de la Cooperativa o la "recuperación de un frigorífico recuperado", con una nueva Comisión Directiva, hoy suman 87 asociados con una producción importante que los condujo a la apertura de una carnicería social y popular en la puerta del predio del frigorífico destinada a la venta de carne al costo para abastecer a los barrios que lo rodean. Actualmente, el frigorífico presta servicios de faena y también puede alquilar sus instalaciones para que faenen otros, ya que tienen matrícula nacional de despostada para llevar carne hasta Tierra del Fuego.

En este caso, como en gran parte de las fábricas recuperadas, el proceso de resistencia y de lucha por no perder los puestos de trabajo produjo la construcción colectiva de un conjunto de prácticas y de saberes nuevos para ellos mismos y una reconfiguración de la subjetividad como trabajador (Coussirat, 2010). En este proceso de movilización y conflicto inicial propio del control obrero de la empresa, el trabajador adquiere una identidad social colectiva, lo que le permite hablar de un nosotros constituido por el proyecto común que los incluye. Si bien el trabajo es el eje articulador del colectivo, este tiene otras características. La organización autogestiva en cooperativas favorece la democratización en la toma de decisiones y la ruptura de la relación tradicional patrón-trabajador. Aparece entonces la figura del trabajador-asociado que tiene el control y el conocimiento de todo el proceso productivo y de comercialización. Estas formas organizativas requieren la constitución de fuertes vínculos con la sociedad para el sostenimiento y la legitimidad de las acciones. Estas relaciones se dan fundamentalmente a través de centros culturales, escuelas populares y actividades solidarias (Cantamutto, 2007; Gómez, 2013).

Estas acciones tienen como fin lograr el apoyo de la comunidad barrial y de la ciudad. En el caso de estudio, la UNS se constituyó en una institución central de articulación, especialmente porque en el tiempo de la recuperación del frigorífico las universidades públicas atravesaban un periodo de sistematización de los programas de extensión. Un porcentaje importante de los proyectos locales se concentraron en los barrios del sur de la ciudad. El equipo de trabajo se encontraba desarrollando el Programa Educativo Arqueología en Cruce en la biblioteca popular "Gabriela Mistral", a dos 
cuadras del Frigorífico. Esta situación permitió el acceso al trabajo a partir de relaciones previamente establecidas y facilitó el vínculo y la participación con la comunidad barrial y especialmente con las y los trabajadores/as del frigorífico. En estas relaciones podemos distinguir dos etapas en función de las acciones políticas que sucedieron en el interior de la empresa. En una primera etapa, el vínculo se estableció con el primer presidente de la Cooperativa de Trabajo, quien manifestó una forma de manejo personalista. En este contexto se realizaron algunas actividades culturales para la comunidad a partir de la planificación del Instituto Cultural de la Municipalidad y se iniciaron los trabajos arqueológicos. Una segunda etapa estuvo marcada por la denuncia de las y los trabajadores/as al primer presidente de la cooperativa como se describió anteriormente. A partir de este momento hubo un cambio en la construcción política por parte de las y los trabajadores/as, que adoptaron una forma horizontal de decisión en estado asambleario. Es en este nuevo contexto que, a partir del año 2015, los trabajadores comenzaron a establecer una relación fluida y de cooperación con la UNS en tres áreas estratégicas: proyecto productivo, proyecto educativo y proyecto cultural. Respecto al primero, graduados de la Licenciatura en Economía de la universidad, asesoraron en distintas etapas de la producción y uno de ellos se incorporó como socio de la cooperativa. Paralelamente a la voluntad de modernizar la producción y venta, los integrantes de la cooperativa manifestaron claramente la necesidad de desarrollar un proyecto educativo, similar al de otras empresas recuperadas. Si bien el proyecto original no pudo ser completado, existe una escuela primaria desde hace tres años y una secundaria desde hace dos, a la que asisten los socios adultos (Visotsky, 2016; Salazar, 2019).

Es en este contexto en el que se inscribe el presente estudio de las percepciones que las y los trabajadores poseen sobre la materialidad, el espacio y la historia de los bienes que tanto desde la academia como de la administración pública se consideran como patrimoniales. Así se planificó, además de los encuentros y observaciones frecuentes, una metodología cualitativa que permitiera que los protagonistas más próximos expresaran sus opiniones en un espacio abierto para el libre intercambio de ideas. En este caso, se organizó en el mes de noviembre del año 2016 una reunión de grupo focal para discutir las percepciones que tenían sobre la zanja, la casa y sus ideas respecto a lo que se podría realizar en torno a los bienes.

Esta es una metodología cualitativa de recolección de información, que tiene un carácter exploratorio y que consiste en la realización de entrevistas colectivas y semiestructuradas en torno a un tema específico que se fundamenta en la epistemología cualitativa, que defiende el carácter constructivo-interpretativo del conocimiento. Posee un lugar relevante en los estudios del campo del patrimonio ya que propicia la toma de decisiones comunitarias, especialmente en lo referido a los modos de intervención sobre los bienes y el espacio patrimonial (Aigneren, 2002; Tomat, 2012, entre otros). En este caso en particular, resulta muy interesante señalar que el proceso de diseño del grupo focal fue participativo, ya que los autores de este trabajo actuaron como moderadores, pero la composición y cantidad de personas participantes fue definida en asamblea. El grupo de entrevistados se constituyó con seis hombres y dos mujeres. Todos ellos mayores de 40 años y con una antigüedad laboral que rondaba en la mayoría en los 20 años, es decir que vivieron el proceso de quiebra y recuperación de la empresa. Muchos de ellos, además, crecieron en el barrio. Esta cierta homogeneidad propuesta, permitió generar un espacio de comunicación relativamente horizontal sensible a las actitudes, experiencias y creencias de los participantes. No obstante, se aseguró la diferencia necesaria en todo proceso de habla. Si bien el grupo se creó para producir una situación discursiva con un carácter aparentemente artificial, reprodujo la forma de relación y acción de las y los trabajadores en estado asambleario. Dadas estas condiciones de diseño, se estableció un conjunto de preguntas abiertas, con un enfoque no directivo, sobre las que se trabajó previamente a la realización de la entrevista, definiendo finalmente un conjunto de siete interrogantes. Todas las preguntas fueron de carácter abierto, de manera que pudieran generar pensamiento crítico y que cada participante tuviera la suficiente flexibilidad para elaborar sus respuestas.

\section{La frontera como articulación del pasado y del presente}

La primera parte de la entrevista grupal fue la presentación de cada participante, que tuvo como eje no solo el nombre y la edad sino la historia laboral en relación con el proceso de la toma y la organización de la cooperativa. El vínculo entre los participantes mayores estuvo dado por compartir gran parte de su vida laboral e incluso en algunos casos su infancia. Esta familiaridad se profundizó en el proceso de toma y recuperación de la empresa y es por eso que algunos de ellos asimilaron las relaciones sostenidas en el frigorífico con las de sus vínculos familiares, haciendo referencia a que " ... una vez que te enganchás con..con INCOB y...sos parte de la familia ..." (VG).

Terminada la presentación, comenzó la charla a partir de la siguiente pregunta: "¿cuáles son los conocimientos que tienen sobre los aspectos históricos de este espacio?" A partir de esta cuestión inicial, la conversación se desarrolló sin prestar demasiada atención a la información a la que hacemos referencia. Para algunos, ocuparse de las cuestiones patrimoniales e históricas fue referido como un lujo que no estaban en condiciones de darse, ya que como señaló uno de los trabajadores: 
"[...] cuando tengamos la fuente de trabajo...ya la caja en orden, tranquila, ya ahí uno va a poder estar más tranquilo para pensar, a ver qué es lo que se puede hacer con eso...juntar los compañeros, hacer una asamblea, decir bueno: ¿qué vamos a hacer con esto y con aquello?". (RL)

Señalaron que cuando llegue ese momento tendrán que recurrir a los profesionales de la universidad, pero "lo que salga, sale de una asamblea", en relación con la decisión final sobre qué funciones darle a estos bienes y espacios. Hubo una coincidencia general de que aún no era el momento, aunque previeron que ese tiempo llegará:

"[...] hoy en día 'tamos' para otra cosa y...bueno, hace muy poquito que está esta comisión y...la gente recién también ahora está viendo muchas cosas que se pueden hacer y bueno y...también, pensar en el futuro que algún día le podamos dar una utilidad a eso, a todo el predio también". (REG)

Todos acordaron que las cuestiones legales en las que se encontraban (y aún hoy) no les permitía dispersar las energías del conjunto, preocupados por atravesar la crisis económica de ese último año, en la que por un lado consolidaron el proceso de producción y venta, pero con una merma de las ganancias debido al aumento de los servicios y la disminución en el consumo de carne. No solo tuvieron que atravesar los requerimientos legales del proceso de expropiación y de la consolidación de la cooperativa, sino también aquellos derivados del proceso judicial por la denuncia que efectuaron al presidente anterior. En distintos momentos de la conversación expresaban que debían esperar, que el futuro les esperaba con proyectos, pero que el presente tenía dificultades, "hay muchas necesidades en el barrio" (REG) y que consolidar la escuela era fundamental para ellos para la vinculación con la comunidad.

A medida que, por las preguntas realizadas, la conversación iba transitando acerca de las historias contenidas en esos referentes materiales, se comenzó a evidenciar que las historias conocidas eran borrosas, alguna vez habían escuchado hablar de la zanja, pero la habían conocido como la "zanja de Alsina" (1876). Respecto a la casa, existía un conjunto mayor de historias, ya que se encuentra en el predio del frigorífico. Algunos, sin estar muy seguros creían que era un monumento histórico. Una de las trabajadoras señaló haberse ocupado de esto y que empezó a interesarse a partir de la intervención de la universidad. Es ella también quien es la primera en dar algunas pautas de los planes futuros de la casa-azotea. A partir de su intervención todos reconocieron que esa casa tiene un valor histórico pero que, como mencionamos, tenían otras prioridades: la fuente de trabajo, "la lucha es la lucha" (RL).
Si bien manifestaron un conocimiento difuso de los hechos del pasado, se pudo observar también que reconocían la importancia histórica del espacio y su vinculación con la lucha obrera:

"[...] Igual lo que tenemos que saber...ser conscientes y saber es que como es una parte tan importante para Bahía Blanca... de la misma manera creo que estaría bueno que en las escuelas...les enseñen lo importante que es INCOB hoy, que es...un...la primer cooperativa recuperada por la cantidad de gente que somos, el hecho de tener esa casa, que los chicos puedan saber cuál es la historia que pasó por ahí, que por lo menos...cuando vos preguntabas si sabíamos algo es porque tampoco en la escuela nos enseñaron de que ese lugar era histórico, y estaría buenísimo que...en las escuelas primarias o secundarias empiecen a contarles la historia de Bahía Blanca como corresponde...y que cuando uno festeja el '11 de Abril' no sea solamente el día de...de Bahía Blanca, que uno no entiende ni que lugares tenemos históricos sino que la casa es importante". (MdCD)

A partir de la palabra de una de las trabajadoras, reconocieron que considerar a estos bienes como patrimoniales, podría acarrear algunos beneficios. Propusieron a futuro una decisión colectiva, entre los profesionales y las y los trabajadores, para establecer qué podría ofrecer cada uno. Es interesante destacar que nunca se observó como un proceso unilateral, producto de una sola persona que tome una decisión, por el contrario, toda resolución es hasta la actualidad, producto de una asamblea.

A partir de este momento, la intervención de los entrevistados comenzó a girar en torno a las posibilidades que podría tener considerar estos bienes como patrimoniales, en relación con su lucha, especialmente en torno a la historia que portan, marcada por el conflicto desigual y como un momento histórico definitorio. Es por eso que a partir de esta situación se fueron articulando los discursos que elaboraron una analogía entre la historia del frigorífico y la de este sector de la ciudad. Ambos poco conocidos, pero ejemplos de lucha de los sectores oprimidos, periféricos en un sentido espacial y social. Esto se tradujo en una necesidad de equiparar la historia del siglo XIX y la historia del frigorífico.

Un tópico que se reiteró una y otra vez fue la ausencia del papel de la escuela en la difusión del conocimiento sobre el pasado y el presente. En definitiva, el discurso de las y los trabajadores está reclamando la presencia del estado. Luego, una vez que la conversación introdujo la importancia patrimonial de estos bienes, algunas intervenciones giraron alrededor de algunas propuestas convocantes para los vecinos, como representaciones 
teatrales sobre aspectos invisibilizados de historia de los pueblos originarios, identidad compartida por algunos de los miembros de la cooperativa. En este punto se produjo un giro en el transcurrir de la conversación y las y los trabajadores tomaron la rienda de la entrevista. Por un lado, comenzaron a emerger las historias de las infancias de aquellos que se criaron en el barrio, sus juegos y sus conocimientos de las historias del lugar. Por otro, se reforzó la analogía como explicación histórica y la concepción del origen, lo fundacional, de la ciudad, pero también de la historia obrera. Es decir, la necesidad de establecer los eventos de una línea histórica, en la que la recuperación del frigorífico necesariamente debería ocupar un lugar en la historia de la ciudad, ya que, como señaló uno de los trabajadores: "...está bien ehhh está bueno contar la historia de la casona, pero también me parece que iría de la mano la historia de INCOB que para Bahía Blanca es, es muy importante..." (VG). En este punto es interesante destacar la autopercepción que tienen sobre su propia lucha equiparándola con la historia de la ciudad. A partir de este momento comenzaron a reconocer la potencialidad de la historia condensada en ese espacio:

“Hay, hay una buena historia acá...ehhh, o sea si te pones a pensar fríamente, para alguien que le interese...ehhh...hay una historia terrible acá... desde la zanja hasta hoy en día, el presente... está en Bahía y mucha gente no lo sabe [...]". (REG)

Incluso, la reafirmación que las y los trabajadores realizaron sobre los sucesos del frigorífico compite con la historia del siglo XIX. Aunque reconocieron que esta, como tantos otros aspectos, también fue ocultada:

"Porque hay lugares ¿viste? que uno va y dice: "ahh por acá pasó...no sé..." ¿viste que hay casas que en Buenos Aires les ponen con reseñas históricas y todo? y después no le das importancia. Pero acá si te pones a pensar, hoy como dijo mi compañera seguís teniendo la misma lucha que tuvieron los de los indios en su momento...o más, porque estamos viviendo en otro siglo donde las cosas tendrían que ser mucho más fáciles pero esta...por algo está la casualidad de la historia...". (MDC)

Un nuevo paralelismo entre la historia de las sociedades indígenas en el siglo XIX y las del siglo XXI pudo observarse cuando manifestaron su modo de acción para recuperar la fuente de trabajo:

"Claro, porque antes era defender con...con armas y era la ley del más fuerte. Hoy en día, no es usar armas, pero tenes que seguir luchando con la burocracia, con toda la política esta...es, es una lucha...no sé si no es peor [risas] esta lucha, te desgasta...yo no sé, pero es lo que a mí me parece, porque esta lucha ehhh, realmente sí, es una lucha que te afecta psicológicamente te...te... te va desgastando". (VG)

Finalmente, los últimos momentos de la entrevista fueron "coordinados" por ellos siendo este espacio utilizado para manifestar la angustia que significó haber atravesado todo el conflicto. Señalaron la inevitabilidad de los sucesos históricos, no eligieron estar allí, se vieron empujados por la necesidad de cobrar una indemnización y esa lucha inicial se convirtió en una forma de vida, en un medio de subsistencia y en una acción política. En esa praxis se definió la asamblea como la metodología que coordina las decisiones en el interior de los muros del frigorífico, que incluyen, entre otras cosas, lo que se quiere y se puede hacer con el patrimonio. Las expresiones vertidas en esta instancia de conversación fueron sostenidas en los últimos tres años. Las condiciones referidas por las y los trabajadores respecto a la crisis económica por la que atravesó el frigorífico, así como las cuestiones legales sin resolver estuvieron presentes, pudiéndose sólo resolver el juicio al primer presidente de la Cooperativa con una sentencia a favor de la actual conducción.

\section{Discusión}

En este trabajo se pretendió, por un lado, historizar el espacio por el frigorífico recuperado INCOB. Esto permitió comprender las diferentes capas de sentidos en el espacio y la constitución del paisaje cultural a partir de su materialidad, desplegando su biografía a través de la cartografía, los documentos y las memorias de miembros de la cooperativa. Por otro lado, se buscó presentar desde un punto de vista histórico las concepciones heterogéneas acerca del patrimonio y los planes y las propuestas de los gestores locales, los investigadores y las y los trabajadores/as. Esta perspectiva mostró las estrategias de los grupos dominantes en la reproducción de distintas versiones de la historia local a lo largo del siglo XX. Las historias de los aficionados de la primera mitad del siglo $X X$ se continuaron en las acciones de la Comisión de Reafirmación Histórica (desde 1976) y en los momentos más cercanos en las acciones de algunos vecinos ligados a la escritura y difusión de la historia de la fundación de la ciudad. Esta trayectoria consolidó una matriz histórica que reprodujo en el siglo $X X$ el binomio civilización-barbarie, realzando la ocupación político militar del espacio fronterizo del sur bonaerense en territorios habitados por las sociedades indígenas. El predominio de estas versiones del pasado difundidas especialmente en el diario local La Nueva Provincia, cambió con el retorno de la democracia. Los gobiernos municipales radicales desde 1984 promovieron una serie de acciones que brindaron herramientas legales y de gestión para la definición, el registro y la conservación del patrimonio que se cristalizaron en la creación de la Comisión para la preservación del Patrimonio 
Arquitectónico, urbanístico e histórica y el Registro Municipal de bienes muebles, inmuebles y áreas de valor patrimonial. En este caso, se cambió el eje de los hechos a resaltar y mientras la primera etapa enfatizó la "primera fundación" de Bahía Blanca, las gestiones municipales a partir de la década de 1980 promovieron el reconocimiento de la llamada "segunda fundación". Como consecuencia, se registraron y declararon como bienes patrimoniales a la materialidad que evidenciaba la modernidad de fines del siglo XIX y principios del XX con foco en el núcleo ferroportuario y la arquitectura institucional y familiar localizada en el centro histórico de la ciudad. Esta perspectiva estuvo fuertemente influenciada por el predominio de los arquitectos en las áreas de decisión y de gestión. Esta matriz histórica prevalece en la actualidad de manera hegemónica en la toma de decisiones de gestión municipal y en las versiones de la historia local que se difunden en el diario, los portales de información y en materiales de comunicación.

Tal como señala Prats (2004), estos repertorios patrimoniales son activados por versiones ideológicas de la identidad, que no son neutrales o inocentes, sean conscientes o no de ello los correspondientes gestores del patrimonio. En el caso de estudio hubo cambios en estas versiones a lo largo del siglo $\mathrm{XX}$, aunque ambas relacionadas con los valores hegemónicos del poder político local. Esto evidencia que no es la sociedad como sujeto colectivo quien activa estos repertorios patrimoniales y no siempre los gestores se ven interesados en promover su participación.

Una práctica participativa del patrimonio, en la que las diferentes versiones de la identidad tengan lugar, pone en jaque algunos de los referentes patrimoniales activados, incluso como en el caso de estudio, el propio concepto de patrimonio. La voz de las y los trabajadores/ as del frigorífico INCOB permiten comprender que las activaciones patrimoniales no necesariamente son compartidas por todos los colectivos sociales, sin que esto signifique que no existan versiones del pasado en juego. En este caso, estas se activaron con la intervención de los arqueólogos que permitieron revelar aspectos no conocidos de un área de la ciudad. Las y los trabajadores /asseñalaron de forma reiterada y enfáticamente que esas versiones del pasado sólo tienen valor en tanto reafirman las versiones de la identidad construida desde las nuevas subjetividades como trabajadores. El protagonismo en la toma de decisiones se evidencia, no solo en lo relativo a la organización de la producción del frigorífico, sino en otros aspectos de la vida cooperativa que incluye la gestión de estos bienes. Especialmente el paralelismo entre la historia de la frontera del siglo XIX y la lucha por la recuperación y el control obrero del frigorífico les permite considerar que estos bienes pueden ser activados como mecanismos simbólicos para legitimar el proceso vivido. Sin embargo, sus tiempos no son los mismos que los de los gestores e incluso de los investigadores. Al momento de finalizar este artículo, aún sigue sin resolverse la situación sobre la propiedad de estos terrenos, esperando un fallo favorable de la Corte Suprema de la Provincia de Buenos Aires para poder avanzar sobre cuestiones como las tratadas en estas páginas.

Bahía Blanca, 19 de febrero de 2020

\section{Agradecimientos}

Queremos expresar nuestro agradecimiento a las y los trabajadores del frigorífico INCOB, quienes diariamente luchan por el mantenimiento de su fuente de trabajo y por condiciones de vida digna para sus familias y para los vecinos del barrio.

\section{Bibliografía}

Acuto F. A. (2013). ¿Demasiados paisajes?: Múltiples teorías o múltiples subjetividades. Anuario de Arqueología, (5), 31-50.

Aigneren, M. (2002). La técnica de recolección de información mediante los grupos focales. La Sociología en sus Escenarios, (6). Recuperado de http://aprendeenlinea.udea.edu.co/revistas/index. php/ceo/article/view/1611/1264

Bayón, C., y Pupio, A. (2003). La construcción del paisaje en el sudoeste bonaerense (1865-1879): una perspectiva arqueológica. En R. Mandrini y C.D. Paz (Comp.), Las fronteras hispanocriollas del mundo indígena latinoamericano en los siglos XVIII-XIX. Un estudio comparativo, (pp.345-374). Tandil, Argentina: Universidad Nacional del Centro de la Provincia de Buenos Aires-Universidad Nacional del Sur.

Bianchi Díaz M.G., y Ockier C.E. (2012). La apropiación de tierras en el asentamiento Villa Nocito (Bahía Blanca, Argentina) por inmigrantes chilenos. Procesos urbanos informales Seminario internacional. Recuperado de https://issuu.com/ sem_proceso_urbanos_informales/docs/02_cla_ apropiaci_n_de_tierras_en_el_asentamiento_

Bloemers, T. (2010). Introduction. En T. Bloemers, H. Kars, A. van der Valk, M. Wijnen (Ed.), The cultural and heritage Paradox. Protection and development of the Dutch Archaeological-Historical Landscape and its European Dimension (pp 3-16). Amsterdam, Países Bajos: Amsterdam University Press.

Borgani, C. (2007). La construcción de la identidad urbana: el análisis de las referencias históricas. Actas de las II Jornadas de Investigación en Humanidades, Universidad Nacional del Sur, Bahía 
Blanca. Recuperado de http://www.humanidades. uns.edu.ar/publicaciones/actas-de-jornadas-deinvestigacion-en-humanidades/entrada/30/IIJornadas-de-Investigacion-en-Humanidades-BahiaBlanca-30-de-agosto-al-1-de-septiembre-de-2007

Cantamutto, F. J. (2007). Empresas recuperadas en el Sur bonaerense: el caso Paloni. En Actas del VII Congreso Argentino Chileno de Estudios Históricos e Integración Cultural, Facultad de Humanidades de la Universidad de Salta, Salta, Argentina, 20 p. Congreso organizado por la Escuela de Historia, Facultad de Humanidades, Universidad Nacional de Salta, Salta, Argentina.

Corimayo H. y Acuto F. (2015). Saber indígena y saber arqueológico en diálogo: interpretando la cultura material diaguita-kallchaquí. En F. Acuto y V. Franco Salvi (Ed.), Personas, cosas, relaciones. Reflexiones arqueológicas sobre las materialidades pasadas y presentes, (pp. 249-247). Quito, Ecuador: Abya-Yala.

Coussirat, M. B. (2010) Re-apropiación de imaginarios sociales (tesis de grado) Universidad Nacional de La Plata, Argentina. Recuperado de: http://www.memoria.fahce.unlp.edu.ar/tesis/ te.569/te.569.pdf

Crespo, C. (2010). Saber arqueológico y memorias locales en la Comarca Andina del Paralelo 42\%, Patagonia (Argentina), Magallania, 38 (1), 71-86.

Diez, M. N. (1999). Cultura Popular e Inmigración: Algunos rasgos de la cultura de los inmigrantes chilenos en Bahía Blanca (1960-1990). Cuadernos del Sur-Historia, (28), 95-123.

Dominella, V. L. (2015). Catolicismo liberacionista y militancias contestatarias en Bahía Blanca: Sociabilidades y trayectorias en las ramas especializadas de Acción Católica durante la efervescencia social y política de los años '60 y '70. (Tesis doctoral). Facultad de Humanidades y Ciencias de la Educación, Universidad Nacional de la Plata, Argentina.

Endere, M. L. (2016). Los retos de la multivocalidad en los nuevos escenarios del patrimonio. En C. García Gianotti, D. Barreiro Martínez y B. Vienni Baptista (coord.), Patrimonio y Multivocalidad. Teoría, práctica y experiencias en torno a la construcción del conocimiento en Patrimonio, (pp.11-16). Montevideo, Uruguay: CSIC.

Flores, C. y F. A. Acuto (2015). Pueblos originarios y arqueología argentina. Construyendo un diálogo intercultural y reconstruyendo la arqueología,
Intersecciones en Antropología, 186, (16), 179-194.

Gómez, A. (2013). Sostenimiento económico de Ias ERT en Argentina. Caso de estudio: cooperativa de trabajo INCOB Ltda. Bahía Blanca. (Tesina de grado). Universidad Nacional del Sur, Bahía Blanca, Argentina.

Guerreiro, H. F. (2011). Los ferrocarriles en Bahía Blanca F.C.B-B.N.O-F.C.B.A.P 1891-1924, (1). Bahía Blanca, Argentina: Edición del autor.

Halbwachs, M. (2004). La memoria colectiva. Zaragoza, España: Prensas Universitarias de Zaragoza.

Haraway, D. (1995). Conocimientos situados: la cuestión científica en el feminismo y el privilegio de la perspectiva parcial. En Ciencia, cyborgs $y$ mujeres. La reinvención de la naturaleza, pp. 313346. Madrid, España: Ediciones Cátedra, Universidad de Valencia.

Jelin, E. (2002). Los trabajos de la memoria. Buenos Aires, Argentina: Siglo XXI.

Kasparian, D. (2013). De alianzas y solidaridades. Las articulaciones no mercantiles en las empresas recuperadas de la Ciudad de Buenos Aires OSERA (8). Recuperado de http://webiigg.sociales.uba.ar/ empresasrecuperadas/PDF/PDF_08/De_alianzas_y_ solidaridades.pdf

Keith, M. y Pile, S. eds. (1997). Geographies of Resistance. Londres, Inglaterra: Routledge.

Kopytoff, I. (1991) La biografía cultural de las cosas: la mercantilización como proceso. En A. Appadurai (Ed.), La vida social de las cosas. Perspectiva cultural de las mercancías (pp. 89-122). México: Grijalbo.

La ciudad conmemoró el 163 aniversario de su fundación (12 de Abril de 1991), La Nueva Provincia, pp. 9.

Mandrini, R. (1992). Indios y fronteras en el área de las pampas (siglo XVI-XIX). Balance y perspectivas. Anuario del IEHS, 7: 59-92.

Millán, M., Chaparro, M.G. y Mariano M. (2019). Diálogos interculturales sobre territorios ancestrales en la provincia de Buenos Aires, Argentina, Iconos. Revista Ciencias Sociales: 161 - 184

Minervino, Mario, (23 de noviembre de 2014). El Congreso analiza su valor. Un sitio histórico y patrimonial que no es. La Nueva Provincia. Recuperado de https://www.lanueva.com/ 
nota/2014-11-23-0-19-0-un-sitio-historico-ypatrimonial-que-no-es

Miravalles, A. (2013). Los talleres invisibles: una historia de los Talleres Ferroviarios Bahía Blanca Noroeste. Bahía Blanca, Argentina: FerroWhite.

Milesi, G.E., y Vázquez R.A. (2013). Búsqueda de los sitios de observación del tránsito de Venus en 1882 en territorio argentino. II. Bahía Blanca. Asociación Argentina de Astronomía, 56, 467-470.

Ockier C.E. (2001). Los procesos de producción y apropiación de la tierra, organización y transformación del espacio en la ciudad de Bahía Blanca. Desde la génesis hasta la realidad urbana actual. (Tesis de doctorado). Universidad Nacional de Cuyo, Mendoza, Argentina.

Ockier, C. E., Bianchi Díaz M.G. y Verdera, G. (2007). Características geo-ambientales de los barrios Maldonado y Villa Nocito: asentamiento de población chilena. Párrafos Geográficos, 6 (1), 100-116.

Pastrana, E. (1965). Reducto Rural Fortificado. Pergamino, Argentina: I.N.T.A.

Peter, B. (1884). Informe sobre las observaciones de Venus practicadas por la Comisión Astronómica Alemana en Bahía Blanca. Boletín de la Academia Nacional de Ciencias (Córdoba), (6), 487-515.

Prats, L. (2004). Antropología y patrimonio. Barcelona, España: Ariel patrimonio.

Prieto, M.B. (2012). Diferenciación Socio-Espacial Urbana. El Caso De Bahía Blanca, Argentina. Revista digital del Grupo de Estudios sobre Geografía y Análisis Espacial con Sistemas de Información Geográfica, 4 (4), 187-214.

Pronsato, D. (1954). Luces de mi tierra. Bahía Blanca, Argentina: Asociación Artistas del Sur de Bahía Blanca.

Puig Domenech, J.A. y Thill, J.P. (2003). Guardias, Fuertes y fortines de la frontera sur. Historia, antecedentes y ubicación catastral. Buenos Aires, Argentina: Servicio Histórico del Ejército.

Puliafito, C. (2007). La Legione Italiana, Bahía Blanca, 1856. El frente olvidado del Risorgimento. Bahía Blanca, Argentina: Edición del autor.

Pupio, A. y H. Perriere (2013). Malones, fortines y estancias en la identidad de General Daniel Cerri. Bahía Blanca, Argentina: EdiuNS.
Ratto, S. y Santilli, D. (2004). De factoría a poblado agropecuario. La evolución del partido de Bahía Blanca hacia 1869. Cuadernos del Sur-Historia, 33, 47-78.

Rebón, J. (2005). Trabajando sin patrón Las empresas recuperadas y la producción. Documentos de trabajo del Instituto de Investigaciones Gino Germani, Facultad de Ciencias Sociales, Universidad de Buenos Aires, Buenos Aires.

Ribas, D. y Tolcachier, F. (2012). La California del Sur: de la construcción del nudo ferro-portuario al centenario local (Bahía Blanca, 1884-1928), Bahía Blanca, Argentina: EdiuNS.

Ricoeur, P. (1995). Tiempo y Narración I. La configuración del tiempo en el relato de histórico. Madrid, España: Siglo XXI.

Rivolta. M.C., Montenegro, M., Menezes Ferreira, L. y Nastri, J. (Ed.) (2014). Multivocalidad y activaciones patrimoniales en Arqueología: perspectivas desde Sudamérica, Buenos Aires, Argentina: Fundación de Historia Natural Félix de Azara.

Salazar, C. (2019) Educación de adultos y educación popular: análisis situado desde la experiencia del Frigorífico Incob. Revista nuestrAmérica, 71-81. Recuperado de https://www.redalyc.org/ jatsRepo/5519/551960420004/

551960420004.pdf.

Salerno, V. (2018). Testimonios que nos da la tierra. Apropiación de objetos arqueológicos en la provincia de Buenos Aires, Argentina. Antípoda. Revista de Antropología y Arqueología, 31, 89-107.

Sather-Wagstaff, J. (2015). Heritage and Memory. En E. Waterton y S. Watson (editores), The palgrave handbook of contemporary heritage research (pp. 191-204). Londres, Inglaterra: Pelgrave Macmillian.

Sesquicentenario de la fundación de Bahía Blanca 1828-1978. Exposición histórica, política social y económica de su evolución.

Tolcachier, F. (2018). Crónica de un derrumbe anunciado. En E. Heredia Chaz (coord.), La Tercera Fundación de Bahía Blanca: la ciudad en la transformación neoliberal (pp. 198-203). Bahía Blanca, Argentina: EdiUNS.

Tomat, C. (2012). El "focus group": nuevo potencial de aplicación en el estudio de la acústica urbana. Athenea Digital, 12 (2), 129-152. 
Tomassini, H. y Vecchi, R. (2015). La "Zanja de Rosas" y el fortín "La Catalina": Un acercamiento inicial a estructuras militares defensivas en Bahía Blanca (primera mitad del siglo XIX). En M. Aguirrezabala, A.M. González Fasani y Tejerina (Eds), Volúmenes Temáticos de las $V$ Jornadas de Investigación en Humanidades (4). Pensar lo local: Visiones y experiencias en torno de la ciudad y su historia (pp. 51-58). Bahía Blanca, Argentina: Hemisferio Derecho.

Tomassini, H. (2017). Arqueología Histórica y activación patrimonial: análisis de estructuras militares defensivas del siglo XIX en Bahía Blanca
(Tesina de grado). Universidad Nacional del Sur, Bahía Blanca, Argentina.

Viñuales, G. y Zingoni J.M. (1990). Patrimonio urbano y arquitectónico de Bahía Blanca. Bahía Blanca, Argentina: La Nueva Provincia, Instituto Argentino de Investigación de Historia de la Arquitectura y del Urbanismo.

Visotsky, J. (2016). Educación de los trabajadores y trabajadoras en INCOB-Industrias de la carne obreras. Proyecto de Extensión Universitaria, Departamento de Humanidades, Universidad Nacional del Sur, manuscrito, inédito. 